\title{
Measuring the Electronic Structure of Nanocrystal Thin Films Using Energy-Resolved Electrochemical Impedance Spectroscopy
}

\section{Journal Article}

Author(s):

Volk, Sebastian (1D; Yazdani, Nuri (10; Sanusoglu, Emir; Yarema, Olesya (i); Yarema, Maksym (1); Wood, Vanessa (i)

Publication date:

2018-03-15

Permanent link:

https://doi.org/10.3929/ethz-b-000252748

Rights / license:

In Copyright - Non-Commercial Use Permitted

Originally published in:

The Journal of Physical Chemistry Letters 9(6), https://doi.org/10.1021/acs.jpclett.8b00109 


\section{Measuring the Electronic Structure of Nanocrystal}

\section{Thin Films using Energy-Resolved Electrochemical Impedance Spectroscopy}

Sebastian Volk, Nuri Yazdani, Emir Sanusoglu, Olesya Yarema, Maksym Yarema, Vanessa

Wood $^{*}$

*Department of Information Technology and Electrical Engineering, ETH Zurich, Gloriastrasse

35, 8092 Zurich, Switzerland

KEYWORDS: density of states, nanocrystal thin films, quantum dots, $\mathrm{PbS}$, ligand, photovoltaics, nanocrystal doping, energy-resolved electrochemical impedance spectroscopy, EIS, ER-EIS 


\section{ABSTRACT:}

Use of nanocrystal thin films as active layers in optoelectronic devices requires tailoring of their electronic band structure. Here, we demonstrate energy-resolved electrochemical impedance spectroscopy (ER-EIS) as a method to quantify electronic structure in nanocrystal thin films. This technique is particularly well-suited for nanocrystal-based thin films as it allows for in-situ assessment of electronic structure during solution-based deposition of the thin film. Using wellstudied lead sulfide nanocrystals as an example, we show that ER-EIS can be used to probe the energy position and number density of defect or dopant states as well as the modification of energy levels in nanocrystal solids that results through the exchange of surface ligands. This work highlights that ER-EIS is a sensitive and fast method to measure the electronic structure of nanocrystal thin films and enables their optimization in optoelectronic devices.

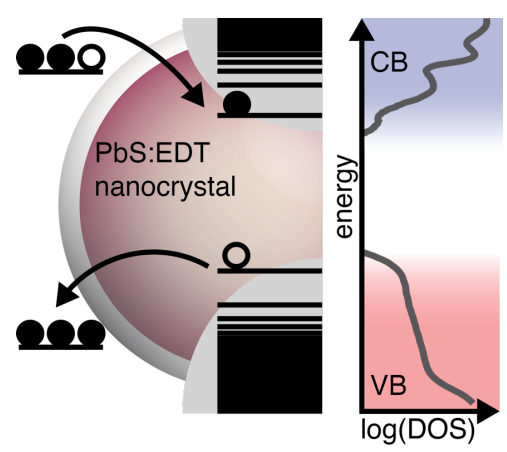


Semiconductor materials derived from colloidally synthesized nanocrystals (NC) are promising candidates for future-generation photovoltaics (PV), transistors, and light-emitting devices. ${ }^{1}$ In NC thin films for optoelectronic applications, short molecules that bind to the NC surface are used to cross-link the NCs into a stable film and facilitate charge transport by tuning the spacing and energy barrier between the individual NCs.,3 Through band engineering using NC layers with different surface treatments ${ }^{4-7}$ as well as $\mathrm{NC}$ surface passivation techniques ${ }^{8}$, record performance metrics such as power conversion efficiencies in NC-based solar cells of up to $13.4 \%$ have been achieved. ${ }^{9}$

Band engineering requires knowledge of the electronic structure of the constituent layers. Ultraviolet photoelectron spectroscopy (UPS), ${ }^{5,10-12}$ kelvin probe spectroscopy (KP), ${ }^{5}$ scanning tunneling spectroscopy (STS), ${ }^{13,14}$ and photoelectron spectroscopy in air (PESA) ${ }^{15}$ have all been applied to measure band onsets in NC solids. However, in UPS, KP, and PESA, only valence band (VB) onsets are determined. The conduction band (CB) onset is estimated by adding the optical or mobility transport gaps or performing inverse photoelectron spectroscopy (IPES). ${ }^{16}$ While STS can be used to extract the density of states (DOS), ${ }^{17}$ it only probes subsections of the $\mathrm{NC}$ ensemble and is often performed at cryogenic temperatures to improve signal to noise ratio. ${ }^{18}$ Because these techniques were developed to work in ultra-high vacuum conditions with pristine surfaces, they are not particularly compatible with solution-processed NC thin films.

Electrochemical techniques, which work by immersing a NC thin film on a conductive substrate into an electrolyte solution, are a more natural choice for measuring $\mathrm{NC}$ thin films that are fabricated by solution compatible techniques. ${ }^{19}$ Cyclic voltammetry (CV), ${ }^{19-22}$ differential pulse voltammetry (DPV),,$^{23,24}$ and electrochemical impedance spectroscopy (EIS) ${ }^{25,26}$ have been applied to measure band onset energies for different types and $\operatorname{sizes}^{27}$ of NCs (in solution and 
incorporated into thin films), and capped with a variety of ligands, ${ }^{13,27,28}$ shell thicknesses in the case of core-shell nanocrystals. ${ }^{29}$ These techniques rely on measurement of the (de-)population of electronic states of the NC thin film in response to modification of the Fermi level, ${ }^{26}$ which is defined by the applied potential. Once the Fermi level rises above unoccupied states in the NC thin film, electrons are injected. Charge transfer to or from the NC thin film (i.e., the electrochemical reduction and oxidation) is measured as a negative or positive current at the counter electrode. Using spectroelectrochemical techniques, Guyot-Sionnest and co-workers ${ }^{30-32}$ and later Boehme et al. ${ }^{33,34}$ showed bleaching of absorption features based upon electrochemical (de-)population through such Fermi level changes. A similar but perturbative approach, potential-modulated absorption spectroscopy (EMAS), has also been applied to determine energy positions of states involved in absorption processes ${ }^{35,36}$ and to investigate photoelectrochemical kinetics at $\mathrm{NP} /$ metal interfaces $^{37}$. However, without a priori knowledge of the corresponding optical matrix element of the underlying process, it is not possible to quantify number densities of states involved in the transition.

Recently, energy-resolved electrochemical impedance spectroscopy (ER-EIS) was presented as a way to quantify the electronic density of states (DOS) of organic polymers. ${ }^{38,39}$ ER-EIS involves sweeping the potential of the thin film to modify the Fermi level, and, at each applied potential $U$, performing impedance spectroscopy (applying a small perturbing potential at different frequencies and analyzing the magnitude and phase of a current response). To obtain the electronic DOS from ER-EIS, one uses the fact that the DOS is defined as the number density of states at a given energy $E$ in an energy interval $d E: g(E)=\frac{d n}{d E}$ and that the current density $j$ between the thin film and electrolyte can be written as $j=e k_{e t} n_{s}[A]$, where $e$ is the elementary charge, $k_{e t}$ is the charge-transfer coefficient, $n_{s}$ is the semiconductor surface carrier 
concentration at the Fermi level, and $[A]$ is the electrolyte concentration. ${ }^{40}$ Application of the perturbing potential varies $n_{s}$, and the charge-transfer resistance $R_{c t}$ associated with this process can be extracted by fitting the EIS data. The $\operatorname{DOS}^{38}$ can therefore be calculated for each applied potential $U$ using:

$g\left(E_{F}=e U\right)=\frac{d n_{S}}{d(e U)}=\frac{1}{e k_{e t}[A] S} \frac{d(j S)}{d(U)}=\frac{1}{e k_{e t}[A] S R_{c t}},[1]$

where $\mathrm{S}$ is the active sample surface area. As discussed by Lewis, ${ }^{40}$ changing the potential at semiconductor electrodes in contact to an electrolyte does not affect the charge-transfer rate $k_{e t}$, so $k_{e t}$, [A], S, and $e$ are all physical constants or constant experimental parameters. Therefore, the DOS is inversely proportional to the charge-transfer resistance measured using ER-EIS.

Here, we show that ER-EIS is applicable to NC thin films, despite the ill-defined surface area compared to a smooth organic polymer thin film with a well-defined solid-electrolyte interface..$^{33,34}$ We begin by highlighting the advantages of ER-EIS over CV-based measurements of electronic structure. The results from both techniques are compared to density functional theory (DFT) calculations on well-established atomistic models for PbS NCs. ${ }^{41,42} \mathrm{We}$ further validate the ER-EIS method by measuring the DOS of lead sulfide (PbS) NC-based thin films fabricated from NCs of different sizes and surface terminations, and bismuth-doped $\mathrm{PbS} \mathrm{NCs}$ that have been reported to show n-type behavior in devices. ${ }^{43}$ These examples underscore that ER-EIS is a reliable, highly sensitive, and quantitative method to measure electronic structure of NC thin films.

First, we compare ER-EIS to CV measurements of electronic structure to emphasize the quantitative nature of ER-EIS. Both techniques use a 3-electrode setup (Figure 1a), with the NC 
thin film as the working electrode (WE), a $\mathrm{Ag} / \mathrm{Ag}^{+}$reference electrode and a Pt wire counter electrode. A solution of $100 \mathrm{mM}$ tetrabutylammonium hexafluorophosphate $\left(\mathrm{TBAPF}_{6}\right)$ in anhydrous acetonitrile is used as the electrolyte. In this first set of experiments, the WE consists of $\mathrm{PbS}$ NCs with a $1^{\text {st }}$ exciton absorption peak at $1.3 \mathrm{eV}$ in solution, which are cross-linked with 1,2-ethanedithiol (EDT) in three dip-coating steps to form a 12-nm-thick film on the ITO substrate. The CV method and measured current density are shown in Figure 1c. Starting at open-circuit potential (OCP), the voltage is ramped at a constant sweep rate of $10 \mathrm{mV} / \mathrm{s}$ in both directions, each time with a fresh sample. The resulting current density in the first sweep OCP -> $+0.5 \mathrm{~V}(\mathrm{OCP}->-2 \mathrm{~V})$ shows clear oxidation (reduction) features at positive (negative) potentials versus the reference electrode, indicative for the depopulation (population) of energetic states in the thin film (for a detailed discussion of multi-cycle CV data, see Supporting Information). ER-EIS is shown in Figure 1d. Measurements are performed at fixed potentials $E_{c}$ with a step width of approximately $5 \mathrm{meV}$ and a frequency-dependent sinusoidal potential perturbation of amplitude $E_{a}=25 \mathrm{meV}$. A Randles circuit is used to fit the resulting EIS curves and determine the series resistance $R_{s}$, which is in series with a parallel arrangement of the double-layer capacitance $\mathrm{C}_{\mathrm{dl}}$ and a faradaic part of the complex impedance, modeled by charge-transfer resistance $R_{c t}$ and a Warburg element $\mathrm{W}$, representing diffusion processes (see inset). The Nyquist diagram of such a circuit resembles a semicircle in the first quadrant, followed by a linear increase with smaller frequencies. The (extrapolated) intersections of the semicircle with $\operatorname{Im}[Z]=0$ are located at $R_{s}$ and $R_{s}+R_{c t}$, respectively. We choose the frequency of the impedance measurements to be logarithmically spaced between $1 \mathrm{kH}$ and $3 \mathrm{~Hz}$, giving rise to the first half of the semicircle, which then is extrapolated to lower frequencies to extract $R_{c t}$, which, as explained above, is inversely proportional to the electronic DOS. 
Setting SHE (Standard Hydrogen Electrode) as the absolute reference point of the electrochemical scale (-4.44 eV versus vacuum) ${ }^{44,45}$ both $\mathrm{CV}$ and ER-EIS data (i.e., $j$ and $\mathrm{R}_{\mathrm{ct}}{ }^{-1}$, respectively) are transformed to the physical scale (Figure 1e). They show excellent qualitative agreement, with distinct features located in the same energy regions.

A major challenge associated with $\mathrm{CV}$ measurements is deciding how to extract the conduction and valence band onsets. While some studies report the onset of reduction and oxidation waves, others report peak positions..$^{27,46-49}$ Typically, arguments in favor of using reduction and oxidation onset potentials are based upon the fact that peak potentials scale with sweep rate and are thus not a precise and reproducible measure if not performed at very low sweep rates. Furthermore, peak extraction is expected to overestimate the real electrochemical bandgap if band onsets consist out of a closely packed series of states. From our CV data, for example, one could identify a conduction band (CB) onset as the intersection of a tangent to the inflection point of the first prominent signal leg and baseline at $-3.78 \mathrm{eV}$, as the inflection itself at $-3.70 \mathrm{eV}$ or even as signal peak position at $-3.64 \mathrm{eV}$. At the valence band (VB), even more complexity is present due to the presence of a broader feature in the onset region. Using the optically-measured bandgap of $1.3 \mathrm{eV}$, one can theoretically argue for a number of different electrochemical band gaps differing by more than $250 \mathrm{meV}$.

The quantitative nature of ER-EIS can provide guidance on how to correctly identify the different peaks since the order of magnitude of the DOS should be known. To learn how to assign the features in the electronic structure without any a priori knowledge or assumptions, we predict the expected DOS using density functional theory (DFT) calculations. The DFTcalculated number density of states in a thiol-terminated $\mathrm{PbS}$ nanocrystal having a bandgap comparable to the thin film under investigation is shown in Figure 2a. To include size 
dispersion, we broaden each individual state through Gaussian distributions with full widths at half maximum matching those to the $\mathrm{NC}$ photoluminescence signal width $\left(\sigma_{\mathrm{Pl}}=62 \mathrm{~nm}\right.$ for the NCs with $1^{\text {st }}$ exciton peak at $1.3 \mathrm{eV}$ ). The DOS superposition of all broadened states serves as a prediction of the DOS (Figure 2a). The CB onset consists of a single state, while the valence band onset is three-fold degenerate (asymmetries in ligand coverage lift this degeneracy). In both cases, the closest neighboring states are more than two standard deviations of the distribution mean apart, resulting in clear features in the DOS. Therefore, the peak positions of these features can be identified as the band onsets.

This first-principles-informed approach is used to identify features in ER-EIS spectra (Figure 2b). We find a distribution with mean at $-3.67 \mathrm{eV}$ vs. vacuum, which we associate with the first CB state. Similarly, the VB is located at $-4.67 \mathrm{eV}$ vs. vacuum. We identify the feature at $190 \mathrm{meV}$ below the $\mathrm{CB}$ as trap states, which were previously measured using Fourier Transform Photocurrent and Thermal Admittance Spectroscopy on NC diodes..$^{50,51}$ Comparing integrated areas of the distributions and assuming one first $\mathrm{CB}$ state per $\mathrm{NC}$, we find that there is 1 trap

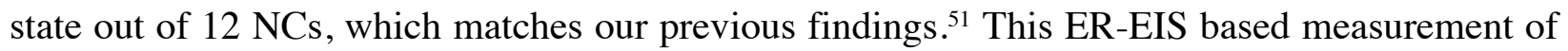
trap state density does not require fabrication and electrical characterization of a functional diode, but measures the NC thin film directly upon fabrication.

We test our approach to characterize the electronic structure of $\mathrm{NC}$ thin films on thin films fabricated from $\mathrm{NC}$ of different sizes having $1^{\text {st }}$ excitation peaks ranging from $0.76-1.54 \mathrm{eV}$. Figure 2c compares the ER-EIS extracted electrochemical bandgaps to the photoluminescence bandgap adjusted for electronic coupling, ${ }^{52}$ which we deduce from the shifts of the $1^{\text {st }}$ excitation feature in absorption spectra between solvated and cross-linked NCs (Supporting Information). The gray dotted line is a guide to the eye and indicates an ideal 1:1 correspondence. Within the 
experimental error bars, which are on average $45 \mathrm{meV}$, we find that both bandgaps scale identically with $\mathrm{NC}$ radius. Note, that the optical bandgap does not include an estimate of the exciton binding energy (EBE). The dielectric constant used to calculate electron-hole coulomb interactions of the form $J_{e-h}=1.786{\frac{e^{2}}{4 \pi \varepsilon_{0} \varepsilon_{Q D} R}}^{10}$ with elementary charge e, vacuum permittivity $\varepsilon_{0}$, dielectric constant of the thin film $\varepsilon_{Q D}$ and NP radius $\mathrm{R}$, is highly dependent on the environment and NP size. Earlier reports ${ }^{10}$ proposed using an optical dielectric constant of $\varepsilon_{\mathrm{QD}}=17.2$, resulting in relatively low EBEs of about $40-100 \mathrm{meV}$ for the NP sizes in this study. For the small NCs (for the largest NC diameter, no clear trap state features are visible), we further find an increasing trap state depth $E_{T}$ with increasing optical bandgap $E_{g}$. We extract a size-dependence of $E_{T}\left(E_{g}\right)=0.5 E_{g}-0.32$, which is in excellent agreement with an earlier study, ${ }^{51}$ having reported a size dependence of $E_{T}\left(E_{g}\right)=0.43 E_{g}-0.31$ (compare gray dashed line; gray shaded area is indicative of the error margin). These results highlight the sensitivity of the ER-EIS measurement approach.

Sensitive measurement of electronic structure is of particular interest for doped-NC thin films. Interstitial as well as substitutional in-situ doping during synthesis have been reported in $\mathrm{PbS},{ }^{43,53-57} \mathrm{PbSe},{ }^{58} \mathrm{InAs},{ }^{59}$ and $\mathrm{CdSe}{ }^{60}$, or via remote dopant in solution, ${ }^{61-63}$ followed by ligandinduced doping.${ }^{64}$ Here, we apply ER-EIS to the example of PbS NCs doped post-synthetically ${ }^{65}$ with 6 at. $\% \mathrm{Bi}$.

Previously, Stavrinadis et al. ${ }^{43}$ employed the combination of UPS and CV to find that in-situ Bidoping changed the $\mathrm{PbS}$ NCs from p-type to n-type behavior with a very broad $\mathrm{CV}$ signal $300 \mathrm{meV}$ below the $\mathrm{CB}$ (associated to a donor state) in the case of $\mathrm{Bi} / \mathrm{Pb}$ doping ratios of $\sim 3.2 \%$ and $1 \mathrm{eV}$ optical bandgap. ${ }^{43}$ We perform ER-EIS on thin films of the PbS NCs before and after post-synthesis doping, and the DOS of both is plotted in Figure 3a. The valence and conduction 
bands onsets overlap $\left(\mathrm{VB}_{\mathrm{PbS}}=4.68 \mathrm{eV}\right.$ and $\mathrm{VB}_{\mathrm{Bi}: \mathrm{Pbs}}=4.70 \mathrm{eV} ; \mathrm{CB}_{\mathrm{PbS}}=3.67 \mathrm{eV}$ and $\mathrm{CB}_{\mathrm{BB}: \mathrm{PbS}}=3.72$ $\mathrm{eV}$ ), which is in agreement with solution absorption spectra that exhibit a $1^{\text {st }}$ exciton peak position at $1.30 \mathrm{eV}$ and $1.31 \mathrm{eV}$ for undoped and doped NCs (Figure 3b) and the shift of the main PL peak by $40 \mathrm{meV}$ from $1056 \mathrm{~nm}$ to $1095 \mathrm{~nm}$ (Figure 3c). The ER-EIS derived DOS for Bi-doped PbS shows a broad feature centered $160 \mathrm{meV}$ below the conduction band, in agreement with expected donor state position from previous studies, exhibiting a number density of $N_{D}=$ $2.2 \cdot 10^{19} \mathrm{~cm}^{-3}$, or approximately $2-3$ dopants per NC (for an estimate of the proportionality factor, linking $g\left(E_{F}\right) \propto R_{c t}{ }^{-1}$, see Experimental Methods). Since the total number of atoms in a $\mathrm{PbS} \mathrm{NC}$ with radius $1.62 \mathrm{~nm}$ and bulk density of $7.6 \mathrm{~g} / \mathrm{cm}^{3}$ is approximately $340,{ }^{66}$ substitutional doping at 6 at $\%$ would theoretically translate into $20 \mathrm{Bi}$ atoms per $\mathrm{NC}$, or 2.5 . $10^{20} \mathrm{~cm}^{-3}$. Therefore not every Bi atom is contributing to the dopant manifold in the NC thin film, which is in agreement with previous findings. ${ }^{43}$ The PL, which after Bi-doping decreases, exhibits a feature at $1415 \mathrm{~nm}$ (indicating a dopant energy at $250 \mathrm{meV}$ below the $\mathrm{CB}$ ). This experiment highlights how a single ER-EIS measurement provides the information that previously needed a combination of electrochemical, photoelectron and optical measurements, and electronic device characterization.

Recent advances in NC photovoltaics use modification of the NC surface to optimize electronic band structure. ${ }^{5,6}$ The intrinsic dipole moments of ligands as well as ligand-NC interface dipoles have been identified as main drivers of the energy level shifts, which are reported to be of up to $0.9 \mathrm{eV}$ for an $1.23 \mathrm{eV}$ band gap $\mathrm{PbS} \mathrm{NC}\left(1^{\text {st }}\right.$ exciton peak). ${ }^{10}$ Here, using the well-studied example of thiol versus halide-terminated NCs, we show that ER-EIS is a tool for measuring the shifts in the CB and VB. 
To identify trends we expect to see, we perform DFT calculations on $\mathrm{PbS}$ NCs with thiol and halide surface termination. For the for $\mathrm{Cl}^{-}, \mathrm{Br}^{-}$and $\mathrm{I}^{-}$treatment, the $\mathrm{CB}$ and $\mathrm{VB}$ onsets shifts to lower energies (from $1.77 \mathrm{eV}$ to $1.97 \mathrm{eV}$ for the $\mathrm{CB}$ and $\mathrm{VB}$ shifts $1.68 \mathrm{eV}$ to $1.8 \mathrm{eV}$ in the case of iodide termination, Figure 4a). Figure $\mathbf{4 b}$ compares ER-EIS measurements of a PbS:EDT crosslinked thin film to halide terminations [tetrabutylammonium bromide (TBABr), tetrabutylammonium iodide (TBABI), tetrabutylammonium chloride (TBACl)]. Relative to EDT, we find an average halide $\mathrm{CB}$ shift of $270 \mathrm{meV}$ and $\mathrm{VB}$ shift of $220 \mathrm{meV}$, with a maximum of $330 \mathrm{meV}$ for the first $\mathrm{I}^{-}$conduction band state. The trends observed in the experiments agree with the DFT, but do not show as dramatic a shift in the energy band structure as predicted by DFT. However, this can be expected because the halide ligand exchange may be incomplete and DFT calculations are performed on charge-neutral idealized systems excluding any interaction with neighboring NCs. This series of measurements highlights how ER-EIS provides an easy and fast method to extract the band shifts that arise as a result of NC surface treatment.

In summary, we introduce ER-EIS as a useful tool to rapidly characterize the electronic structure of NC thin films in parallel with their fabrication. We show that the size and surface termination dependent bandgap energies measured with ER-EIS are in excellent agreement with those found using other electrochemical techniques such as CV, but that ER-EIS paired with structural information of the film is also quantitative and provides the sensitivity to determine the energy position and number density of mid-gap trap states or dopants. 


\section{Experimental Methods:}

NC synthesis: Synthesis of $\mathrm{PbS}$ nanocrystals was carried out according to previously published recipe..$^{5}$ Briefly, the injection mixture was prepared in the air-free glove box by mixing bis(trimethylsilyl)sulphide $(0.639 \mathrm{~mL})$ and dried 1-octadecene $30 \mathrm{~mL})$. Reaction mixture, consisted out of $\mathrm{PbO}(1.35 \mathrm{~g})$, oleic acid $(4.5 \mathrm{~mL})$ and 1-octadecene $(54 \mathrm{~mL})$, was placed in the three-neck flask and heated to $80^{\circ} \mathrm{C}$ under vacuum for $10 \mathrm{~h}$ along with magnetic stirring. During this time, $\mathrm{PbO}$ reacted with oleic acid forming $\mathrm{Pb}$-oleate, which was indicated by complete dissolution of yellow precipitates. Afterwards, reaction mixture was heated to $110^{\circ} \mathrm{C}$ under nitrogen atmosphere, at which conditions, injection mixture was swiftly added, initiating the fast formation of $\mathrm{PbS}$ nanocrystals. After injection, the heating element was displaced and reaction mixture was left for 40 min to gradually cool down to room temperature. $\mathrm{PbS}$ nanocrystals were transferred to glovebox and purified from organic by-products by adding $150 \mathrm{~mL}$ of anhydrous acetone and centrifuging at $6000 \mathrm{rpm}$ for $5 \mathrm{~min}$. Obtained $\mathrm{PbS}$ precipitates were dissolved in toluene, forming long-term stable colloidal solution. Size of PbS nanocrystals was regulated by adjusting amount of oleic acid and growth temperature.

Nanocrystal doping: Colloidal PbS nanocrystals were doped by Bismuth according to a previously published procedure. ${ }^{65}$ Briefly, to prepare 6 at. \% Bismuth-doped $\mathrm{PbS}$ nanocrystals, Bismuth acetate $(2.4 \mathrm{mg})$ was loaded to the three-neck flask along with the mixture of oleic acid $(2 \mathrm{~mL})$ and 1 -octadecene $(4 \mathrm{~mL})$ at $95^{\circ} \mathrm{C}$ under vacuum for $15 \mathrm{~h}$. During this time, Bismuth acetate was completely dissolved and the precursor of Bismuth was subsequently put under nitrogen atmosphere and $90^{\circ} \mathrm{C}$. At these conditions $100 \mathrm{mg}$ of $\mathrm{PbS} \mathrm{NC}$ in toluene were added to the bismuth precursor mixture and after the heating mantle was displaced, the three-neck flask was left to cool down to room temperature. Bismuth-doped PbS NC were transferred to glovebox 
and purified by adding $30 \mathrm{~mL}$ of anhydrous acetone, centrifuging at $6000 \mathrm{rpm}$ for $5 \mathrm{~min}$, and dissolving precipitates in anhydrous toluene (this washing cycle was carried out 2 times in total). Solutions of Bismuth-doped PbS nanocrystals were stored the glove box.

Substrate preparation: ITO substrates were cleaned following a series of sonication cycles $(5 \mathrm{~min}$ each) in the following order: detergent (2 times), DI-water (2 times), Acetone (2 times), Isopropanol (at $140^{\circ} \mathrm{C}$ ). Electrical connection legs were purchased from Ossilla and clipped onto the substrate. Silverpaste was applied at the contact spot to improve electrical connection and dried in an oven for $30 \mathrm{~min}$ at $100^{\circ} \mathrm{C}$. ITO substrates were partially covered with PDMS for three reasons: 1) mechanical stability of the substrate-connector system, 2) definition of the active substrate area and 3) preventing electrical contact between the connection legs and electrolyte. Active areas were defined to $1 \mathrm{~cm}^{2}( \pm 5 \%)$. PDMS curing was performed in an oven at $100^{\circ} \mathrm{C}$ for 1 hour. Samples were then transferred to a nitrogen glovebox and stored until usage.

$N C$ thin film fabrication: Thin films deposition was performed in a nitrogen atmosphere in a glove box. An in-house designed automated dipcoater setup performed a series of dip coating steps consisting of: 1) immersion into NC solution, 2) cross linking step and 3) rinsing step. NCs for the dipcoating process were dispersed in a $5 \mathrm{mg} / \mathrm{ml}$ solution in anhydrous hexane. For a surface ligand exchange to EDT, we used a $5 \mathrm{mM}$ solution of 1,2-ethanedithiol in anhydrous acetonitrile. For halide termination, we used $100 \mathrm{mM}$ solutions of either species: tetrabutylammonium iodide, tetrabutylammonium chloride and tetrabutylammonium bromide in anhydrous methanol. Rinsing was performed in pure anhydrous acetonitrile (anhydrous methanol) for EDT (halide) treatment. For complete electrode coverage, three dipcoating cycles were performed. 
Electrochemical measurement: Standard three-electrode setups were used with a BioLogic RE-7 non-aquaeous reference electrode $\left(\mathrm{Ag} / \mathrm{Ag}^{+}\right)$with an electrochemical potential of $0.542 \mathrm{mV}$ vs. NHE (Normal Hydrogen Electrode), ${ }^{67}$ setting it to $-4.98 \mathrm{eV}$ vs vacuum (assuming similar absolute potentials for SHE and NHE). Tetrabutylammonium hexafluorophosphate in anhydrous acetonitrile served as a supporting electrolyte. In a series of weakly-coordinating anions, TBAPF6 has shown to be the least invasive compared to tetrabutylammonium perchlorate (TBAP) and tetrabutylammonium tetrafluoroborate (TBABF4). To exclude artifacts resulting from previous measurements, every measurement was performed on a fresh sample using a fresh supporting electrolyte. All measurements were controlled by a BioLogic SP-150 potentiostat. CV measurements were performed starting at OCP into either direction with a sweep rate of $10 \mathrm{mV} / \mathrm{s}$. Impedance measurements were performed at frequencies between $1 \mathrm{kHz}$ and $3 \mathrm{~Hz}$ with two measurement points per decade in logarithmic spacing, resulting in a first half semi-circle in the first quadrant in a Nyquist diagram representation. Note, that the chosen frequency range is expected to be material-dependent and might need to be adapted if other than $\mathrm{PbS}$ thin-film systems are under investigation. Starting at OCP, the typical step width between subsequent measurements is $5 \mathrm{meV}$. With a single impedance sweep duration of about $2 \mathrm{~s}$, we estimate the effective potential sweep velocity during the whole experiment at $2.5 \mathrm{mV} / \mathrm{s}$, which is typically regarded to be slow enough to allow the system reaching an equilibrium state, if compared to common sweep rates in CV experiments. ER-EIS measurements were performed on two fresh samples each towards positive and negative potentials versus OCP to calculate mean and standard deviations of $E_{g}$ and $E_{\mathrm{T}}$ in Fig. 2c)-d). For the NC with $1^{\text {st }}$ exciton features at $1.19 \mathrm{eV}$ and $1.31 \mathrm{eV}$, only one trace towards $\mathrm{CB}$ had been available and $55 \mathrm{meV}$ (equivalent to the largest 
standard deviation of the other dots) manually added to account for typical experimental uncertainties.

Quantifying the DOS: An estimate of the proportionality factor, which links $g\left(E_{F}\right) \propto R_{c t}{ }^{-1}$ to provide a quantitative measure of dopant densities, was provided. As the electrolyte can infill the porous nanostructured network, the surface $S$ was no longer restricted to the geometric surface area of the electrode but scaled with the material amount being deposited onto the substrate. Indeed, we found a linear increase in integrated CB state distribution and sample thickness. Such an integrated distribution reflects the total number of probed $\mathrm{CB}$ onset states in the total film. Expecting one such state per $\mathrm{NC}$, we reduced the problem to $N P$ density $=$ $\int_{\text {first } C B \text { state }} g(E) d E=c_{E I S} \int_{\text {first } C B \text { state }} R_{c t}{ }^{-1} d E$, with $\quad c_{E I S} \quad$ including all potentialindependent prefactors. To calculate the NC density, we assumed a BCC arrangement of NC spheres with an interparticle spacing of $1.43 \mathrm{~nm}$, measured through small angle X-ray scattering on PbS:EDT NCs with a diameter of $3.1 \mathrm{~nm}$ (see Supporting Information). This was in agreement with literature, ${ }^{68}$ reporting a $1.6 \mathrm{~nm}$ spacing for a $\mathrm{PbS} \mathrm{NC}$ radius of $1.62 \mathrm{~nm}$. With such a nanoparticle density of $9.8 \cdot 10^{18} \mathrm{~cm}^{-3}$, we derived $c_{E I S}\left(E_{g=1.3 \mathrm{eV}}\right)=1.9$. $10^{24} \Omega(\mathrm{eV})^{-1} \mathrm{~cm}^{-3}$. The complete set of size-dependent prefactors can be found in the

\section{Supporting Information.}

DFT calculations: The PbS NCs were constructed following the atomistic model proposed by Zherebetskyy et al. ${ }^{42}$ with a radius of $\mathrm{r}=\sim 1.2 \mathrm{~nm}$. Bulk rocksalt $\mathrm{PbS}$ (with a $\mathrm{Pb}$ centered on the origin) is cut along the eight [111] planes and six [100] planes. The [111]-surface terminating S atoms are then replaced with the desired ligand. For an intrinsic semiconductor NC,

$$
2 N_{P b}-2 N_{S}+N_{L} V_{L}+N_{c h} e=0
$$


where $N_{x}$ refers to the number of $\mathrm{Pb}$ or $\mathrm{S}$ atoms, ligands $(L)$, or surplus/deficiency of additional charges (ch), and $V_{L}$ is the valence of the ligand. To satisfy eq. (2) and eq. (3), two Pb-ligand pairs were randomly removed, resulting in a NC with $N_{P b}=199$ lead atoms, $N_{S}=140$ sulfur atoms, and $N_{L}=118$ ligands. To capture the chemistry of the $\mathrm{Pb}-\mathrm{S}$ bond present in thiol-terminated NCs, methane-thiol (mth) ligand is selected.

Geometry optimization, and electronic structure calculations are performed within the CP2K program suite utilizing the quickstep module. ${ }^{69}$ Calculations use a dual basis of localized Gaussians and plane-waves, ${ }^{70}$ with a 300Ry plane-wave cutoff. As in previous calculations for $\mathrm{CdSe}^{71}$ and $\mathrm{PbS}^{72} \mathrm{NCs}$, Double-Zeta-Valence-Polarization (DZVP) ${ }^{73}$ Goedecker-Teter-Hutter pseudopotentials $^{74}$ for core electrons, and the Perdew-Burke-Ernzerhof (PBE) exchange correlation functional are used for all calculations. Convergence to $10^{-8}$ in Self-Consistent Field calculations is always enforced. Non-periodic boundary conditions are employed for both the structure and the potential, which is solved using the Wavelet Poisson solver. ${ }^{75,76} 4 \mathrm{nmx} 4 \mathrm{nmx} 4 \mathrm{~nm}$ cubic unit cells are defined for the $r=1.2 \mathrm{~nm}$ NCs. Geometry optimization is performed with the Quickstep module utilizing a Broyden-Fletcher-Goldfarb-Shannon (BFGS) optimizer. A maximum force of $24 \mathrm{meV}^{-1}$ is used as convergence criteria. All atoms in all systems are relaxed. Although the bandgap extracted from DFT for a certain NC size is typically underestimated, the energy distance between neighboring states within the bands are assumed to be realistic. ${ }^{77}$ 
a)

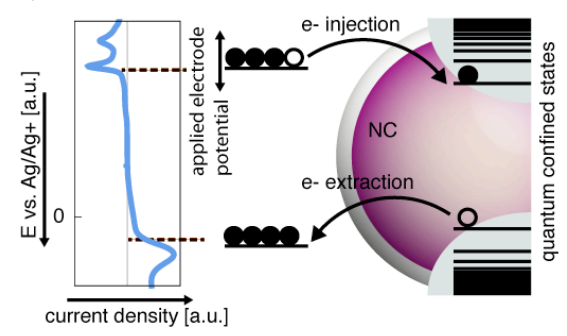

b)

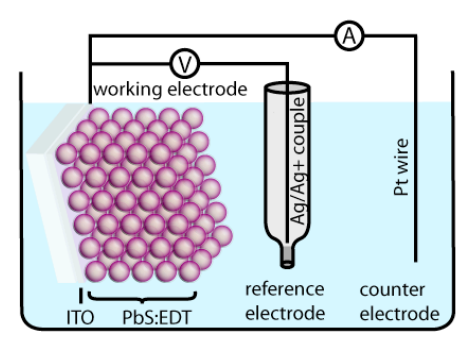

c)

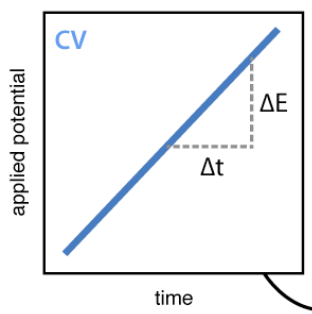

d)

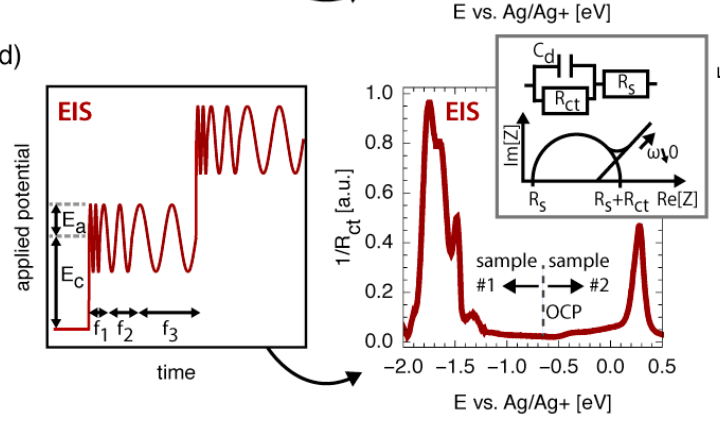

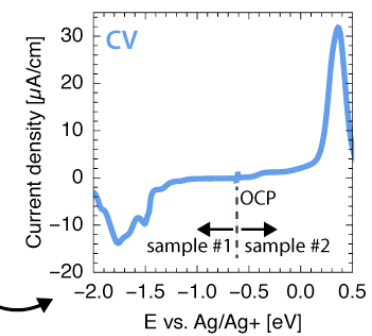

e)

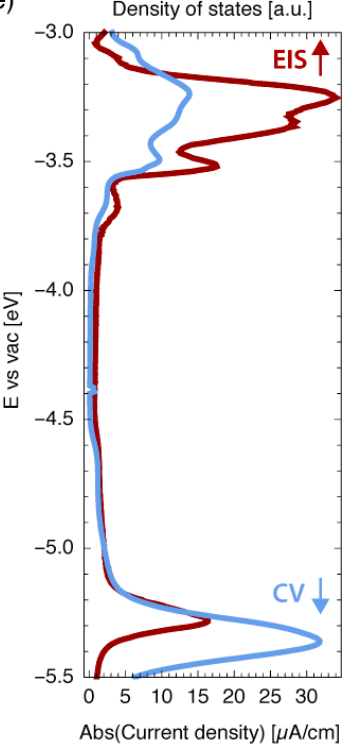

Figure 1. Electrochemical techniques to map the electronic structure in nanocrystal thin

films. a) Schematic of a three-electrode setup in which a nanocrystal thin film deposited on ITO serves as the working electrode (WE). The Fermi level $\left(\mathrm{E}_{\mathrm{F}}\right)$ in the nanocrystal thin film is modified by controlling the electrode potential versus an $\mathrm{Ag} / \mathrm{Ag}+$ reference electrode. Chargetransfer processes are monitored using a Pt counter electrode. b) Schematic of charge-transfer processes based upon electrochemical modification of the Fermi level. Thin film oxidation (or reduction) takes place once the $\mathrm{E}_{\mathrm{F}}$ moves below (or above) the valence (or conduction) band onset, leading to a positive (or negative) current. c) In CV, the potential is varied at a constant rate while measuring the resulting current density. Right: CV experiments are conducted on PbS:EDT thin-films. Starting at open-circuit potential (OCP), consecutive sweeps into positive and negative potential directions are performed on fresh samples to prevent signal distortion by hysteresis (see in-depth discussion in Supporting Information). d) In energy-resolved electrochemical impedance spectroscopy (ER-EIS), the frequency-dependent impedance $Z\left(E_{c}, f\right)$ at a series of applied potentials $E_{c}$ is derived through the magnitude and phase of a current $I$ in response to a small perturbation $E_{a}$. By modeling the complex impedance $Z=Z$ ' $+i Z$ ' by a 
Randles circuit (inset), we extract the charge-transfer resistance $\mathrm{R}_{\mathrm{ct}}$, which is proportional to the density of states. e) The current density in cyclic voltammetry is in excellent qualitative agreement with $\mathrm{R}_{\mathrm{ct}}{ }^{-1}$, extracted out of ER-EIS. Both traces are transferred to the physical scale with zero representing vacuum. 

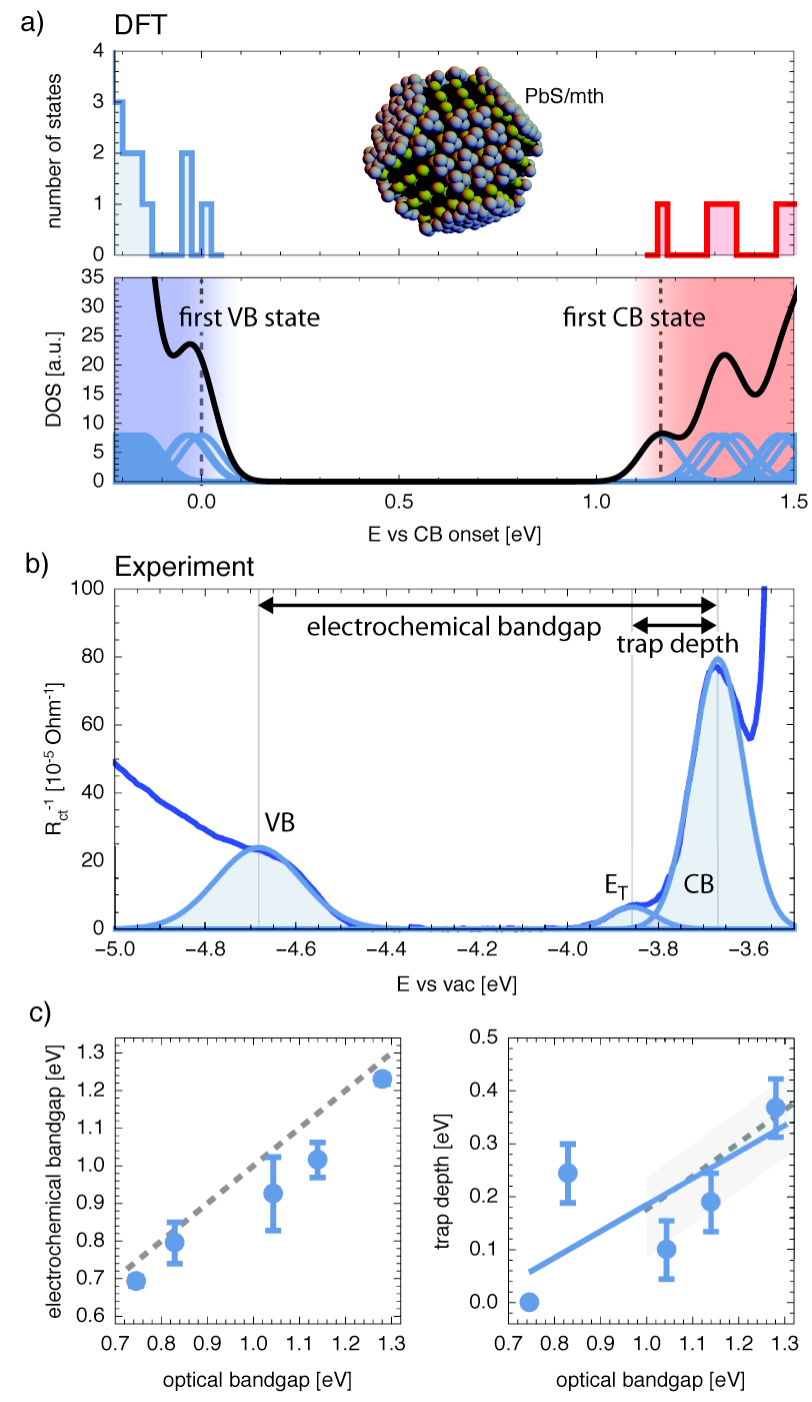

Figure 2. Understanding the density of states in nanocrystal thin films. a) Upper: Densityfunctional theory (DFT) calculations on $\mathrm{PbS}$ nanocrystals with [111]-facets covered with methanethiol (mth) predict a sparse distribution of states in conduction (CB) and valence band (VB) onset regions and a defect-free bandgap. Asymmetry in surface ligand coverage lifts a potential degeneracy at the band onsets. Lower: The expected density of states for a NC ensemble comes from the sum of broadened individual states to account for size dispersion. b) For quantitative insight into the electronic bandstructure, we plot inverse charge-transfer resistance $\mathrm{R}^{-1}$, which is expected to be proportional to the density of states, versus energy. The 
sparse distribution of states enables us to reliably identify the onset of the conduction and valence bands as the means of the distributions. A trap state manifold $\mathrm{E}_{\mathrm{T}}$ at $190 \mathrm{meV}$ below the conduction band is identified. Energy positions of $\mathrm{CB}$ and $\mathrm{VB}$ onsets together with $\mathrm{E}_{\mathrm{T}}$ are indicated (gray line) c) Verification of our interpretation of the ER-EIS data using a series of thin films with NCs of different sizes. Left: The electrochemical bandgap extracted from ER-EIS agrees with the optical bandgap. The gray dotted line shows a 1:1 relation as a guide to the eye. Right: ER-EIS identified trap depths are in excellent agreement with existing literature. For comparison, the NC size-dependent trap depth reported in an earlier study ${ }^{51}$ based upon thermal admittance spectroscopy on devices is plotted with a gray dotted line (error margin in gray background). 

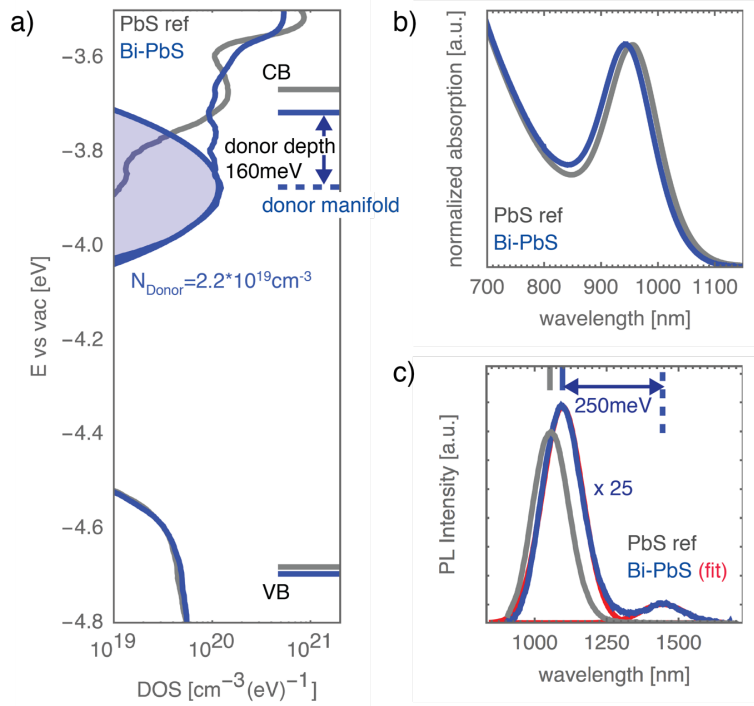

Figure 3. Observation of nanocrystal doping. a) The ER-EIS derived density of states (DOS) for post-synthesis Bi-doped nanocrystal (NC) thin film (blue trace) is compared to undoped NC reference films (gray trace). Whereas the DOS of both doped and undoped material sets perfectly matches in their VB region, the Bi-doping introduces a signal feature at a depth of $160 \mathrm{meV}$ below the conduction band which we assign to a donor manifold. Assuming a Gaussian distribution of donor states (blue shaded curve), we estimate a total number density of 2.2 . $10^{19} \mathrm{~cm}^{-3}$, translating into 2-3 donor states per NC. Extracted band onsets and donor manifold energy positions are indicated at the right-hand side. b) Optical absorption measurements on thin film show a $17 \mathrm{meV}$ redshift in the $1^{\text {st }}$ exciton feature. c) Photoluminescence main peak of the $\mathrm{Bi}$ doped $\mathrm{PbS}$ NCs decreases by a factor of 25 and a small signal feature at higher wavelengths emerges that is linked to the donor state to valence band transition. 

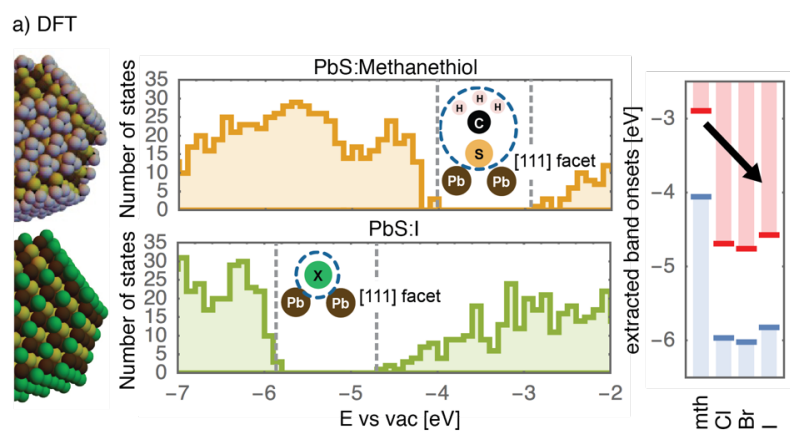

b) EIS
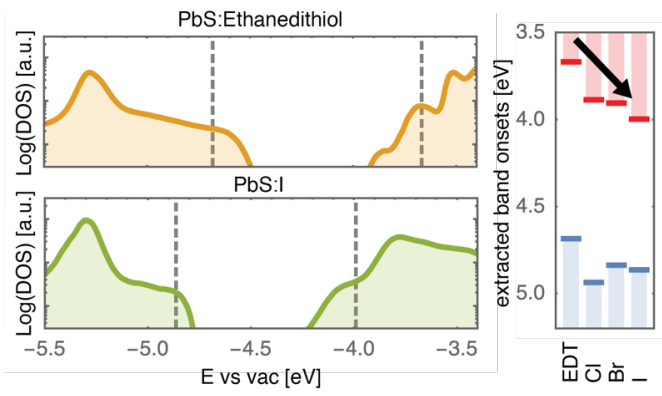

Figure 4. Influence of surface functionalization on the electronic structure a) Density functional theory (DFT) calculations show that, relative to a thiol-based surface termination (methanethiol $=$ mth), halide-terminations (Iodide, Chloride, or Bromide) lower conduction badn (CB) and valence band (VB) onsets by up to $1.9 \mathrm{eV}$. The position of the first $\mathrm{CB}$ and VC state is indicated (gray, dahed line). b) Band onsets measured by ER-EIS. Left: Thiol-based surface termination (orange) is compared to Iodide surface treatment, highlighting the global shift of the bandstructure. Extracted CB and VB onsets are indicated (gray, dashed line). Right: Compared to a EDT surface termination, halide treatments on real nanoparticle networks lower band onsets by up to $330 \mathrm{meV}$ (CB, Iodide termination) in agreement with literature ${ }^{10}$ and following the same trend as the DFT calculations. 


\section{Associated Content:}

Supporting Information: Optical photoluminescence and absorption data as a function of NC size; normalization of density of states to determine number of trap state per NC as a function of NC size; approach to normalize density of states for NC films.

\section{Author Information:}

Corresponding author: *Email: vwood@ethz.ch

Author Contributions: SV fabricated thin films and performed all experiments. NY performed DFT calculations. OY and MY synthesized the material. E.S. performed SAXS experiments. SV and VW envisioned the experiments. The manuscript was written through contributions of all authors.

Notes: The authors declare no financial interest.

\section{Acknowledgements}

The authors gratefully acknowledge funding from the Swiss National Science Foundation through Sinergia (project CRSII2_147615) and the NCCR Quantum Science and Technology. 


\section{References}

(1) Kagan, C. R.; Lifshitz, E.; Sargent, E. H.; Talapin, D. V. Building Devices from Colloidal Quantum Dots. Science. 2016, 353 (6302).

(2) Boles, M. A.; Ling, D.; Hyeon, T.; Talapin, D. V. The Surface Science of Nanocrystals. Nat. Mater. 2016, 15, 141-153.

(3) Kovalenko, M. V.; Manna, L.; Cabot, A.; Hens, Z.; Talapin, D. V.; Kagan, C. R.; Klimov, V. I.; Rogach, A. L.; Reiss, P.; Milliron, D. J.; et al. Prospects of Nanoscience with Nanocrystals. ACS Nano 2015, 9 (2), 1012-1057.

(4) Chuang, C. M.; Brown, P. R.; Bulović, V.; Bawendi, M. G. Improved Performance and Stability in Quantum Dot Solar Cells through Band Alignment Engineering. Nat. Mater. 2014, 13, 796-801.

(5) Ning, Z.; Voznyy, O.; Pan, J.; Hoogland, S.; Adinolfi, V.; Xu, J.; Li, M.; Kirmani, A. R.; Sun, J.-P.; Minor, J.; et al. Air-Stable N-Type Colloidal Quantum Dot Solids. Nat. Mater. 2014, $13(8), 822-828$.

(6) Brown, P. R.; Lunt, R. R.; Zhao, N.; Osedach, T. P.; Wanger, D. D.; Chang, L.; Bawendi, M. G.; Bulović, V. Improved Current Extraction from $\mathrm{ZnO} / \mathrm{PbS}$ Quantum Dot Heterojunction Photovoltaics Using a MoO3 Interfacial Layer. Nano Lett. 2011, 29552961.

(7) Santra, P. K.; Palmstrom, A. F.; Tanskanen, J. T.; Yang, N.; Bent, S. F. Improving Performance in Colloidal Quantum Dot Solar Cells by Tuning Band Alignment through 
Surface Dipole Moments. J. Phys. Chem. C 2015, 119 (6), 2996-3005.

(8) Lan, X.; Voznyy, O.; Kiani, A.; García De Arquer, F. P.; Abbas, A. S.; Kim, G. H.; Liu, M.; Yang, Z.; Walters, G.; Xu, J.; et al. Passivation Using Molecular Halides Increases Quantum Dot Solar Cell Performance. Adv. Mater. 2016, 28 (2), 299-304.

(9) Sanehira, E. M.; Marshall, A. R.; Christians, J. A.; Harvey, S. P.; Ciesielski, P. N.; Wheeler, L. M.; Schulz, P.; Lin, L. Y.; Beard, M. C.; Luther, J. M. Enhanced Mobility CsPbI3 Quantum Dot Arrays for Record-Efficiency, High-Voltage Photovoltaic Cells. Sci. Adv. 2017, $3(10), 1-8$.

(10) Brown, P. R.; Kim, D.; Lunt, R. R.; Zhao, N.; Bawendi, M. G.; Grossman, J. C.; Bulović, V. Energy Level Modification in Lead Sulfide Quantum Dot Thin Films through Ligand Exchange. ACS Nano 2014, 8 (6), 5863-5872.

(11) Crisp, R. W.; Kroupa, D. M.; Marshall, A. R.; Miller, E. M.; Zhang, J.; Beard, M. C.; Luther, J. M. Metal Halide Solid-State Surface Treatment for High Efficiency PbS and PbSe QD Solar Cells. Sci.Rep. 2015, 5, 9945.

(12) Rastogi, P.; Palazon, F.; Prato, M.; Di Stasio, F.; Krahne, R. Enhancing the Performance of CdSe/CdS Dot-in-Rod Light Emitting Diodes via Surface Ligand Modification. ACS Appl. Mater. Interfaces 2018, 10, 5665-5672.

(13) Soreni-Harari, M.; Yaacobi-Gross, N.; Steiner, D.; Aharoni, A.; Banin, U.; Millo, O.; Tessler, N. Tuning Energetic Levels in Nanocrystal Quantum Dots through Surface Manipulations. Nano Lett. 2008, 8 (2), 678-684. 
(14) Steiner, D.; Dorfs, D.; Banin, U.; Sala, F. D.; Manna, L.; Millo, O. Determination of Band Offsets in Heterostructured Colloidal Nanorods Using Scanning Tunneling Spectroscopy. Nano Lett. 2008, 8 (9), 2954-2958.

(15) Jasieniak, J.; Califano, M.; Watkins, S. E. Size-Dependent Valence and Conduction BandEdge Energies of Semiconductor Nanocrystals. ACS Nano 2011, 5 (7), 5888-5902.

(16) Katsiev, K.; Ip, A. H.; Fischer, A.; Tanabe, I.; Zhang, X.; Kirmani, A. R.; Voznyy, O.; Rollny, L. R.; Chou, K. W.; Thon, S. M.; et al. The Complete in-Gap Electronic Structure of Colloidal Quantum Dot Solids and Its Correlation with Electronic Transport and Photovoltaic Performance. Adv. Mater. 2014, 26 (6), 937-942.

(17) Liljeroth, P.; Jdira, L.; Overgaag, K.; Grandidier, B.; Speller, S.; Vanmaekelbergh, D. Can Scanning Tunnelling Spectroscopy Measure the Density of States of Semiconductor Quantum Dots? Phys. Chem. Chem. Phys. 2006, 8 (33), 3845.

(18) Banin, U.; Millo, O. Tunnelling and Optical Spectroscopy of Semiconductor Nanocrystals. Annu. Rev. Phys. Chem. 2003, 54 (1), 465-492.

(19) Liu, J.; Yang, W.; Li, Y.; Fan, L.; Li, Y. Electrochemical Studies of the Effects of the Size, Ligand and Composition on the Band Structures of CdSe, CdTe and Their Alloy Nanocrystals. Phys. Chem. Chem. Phys. 2014, 16 (10), 4778-4788.

(20) Dissanayake, D. M. N. M.; Lutz, T.; Curry, R. J.; Silva, S. R. P. Measurement and Validation of PbS Nanocrystal Energy Levels. Appl. Phys. Lett. 2008, 93 (4), 91-94.

(21) Haram, S. K.; Quinn, B. M.; Bard, A. J. Electrochemistry of CdS Nanoparticles: A 
Correlation between Optical and Electrochemical Band Gaps. J. Am. Chem. Soc. 2001, $123,8860-8861$.

(22) Kucur, E.; Riegler, J.; Urban, G. A.; Nann, T. Determination of Quantum Confinement in CdSe Nanocrystals by Cyclic Voltammetry. J. Chem. Phys. 2003, 119 (4), 2333-2337.

(23) Bloom, B. P.; Zhao, L. Bin; Wang, Y.; Waldeck, D. H.; Liu, R.; Zhang, P.; Beratan, D. N. Ligand-Induced Changes in the Characteristic Size-Dependent Electronic Energies of CdSe Nanocrystals. J. Phys. Chem. C 2013, 117 (43), 22401-22411.

(24) Sobrova, P.; Ryvolova, M.; Hubalek, J.; Adam, V.; Kizek, R. Voltammetry as a Tool for Characterization of CdTe Quantum Dots. Int. J. Mol. Sci. 2013, 14 (7), 13497-13510.

(25) Wang, H.; Wang, Y.; He, B.; Li, W.; Sulaman, M.; Xu, J.; Yang, S.; Tang, Y.; Zou, B. Charge Carrier Conduction Mechanism in PbS Quantum Dot Solar Cells: Electrochemical Impedance Spectroscopy Study. ACS Appl. Mater. Interfaces 2016, 8, 18526-18533.

(26) Bisquert, J.; Fabregat-Santiago, F.; Mora-Seró, I.; Garcia-Belmonte, G.; Barea, E. M.; Palomares, E. A Review of Recent Results on Electrochemical Determination of the Density of Electronic States of Nanostructured Metal-Oxide Semiconductors and Organic Hole Conductors. Inorganica Chim. Acta 2008, 361 (3), 684-698.

(27) Querner, C.; Reiss, P.; Sadki, S.; Zagorska, M.; Pron, A. Size and Ligand Effects on the Electrochemical and Spectroelectrochemical Responses of CdSe Nanocrystals. Phys. Chem. Chem. Phys. 2005, 7 (17), 3204-3209.

(28) Aldakov, D.; Querner, C.; Kervella, Y.; Jousselme, B.; Demadrille, R.; Rossitto, E.; Reiss, 
P.; Pron, A. Oligothiophene-Functionalized CdSe Nanocrystals: Preparation and Electrochemical Properties. Microchim. Acta 2008, 160 (3), 335-344.

(29) Amelia, M.; Avellini, T.; Monaco, S.; Impellizzeri, S.; Yildiz, I.; Raymo, F. M.; Credi, A. Redox Properties of CdSe and CdSe-ZnS Quantum Dots in Solution. Pure Appl. Chem. 2010, $83(1), 1-8$.

(30) Guyot-Sionnest, P.; Wang, C. Fast Voltammetric and Electrochromic Response of Semiconductor Nanocrystal Thin Films. J. Phys. Chem. B 2003, 107 (30), 7355-7359.

(31) Wehrenberg, B. L.; Guyot-Sionnest, P. Electron and Hole Injection in PbSe Quantum Dot Films. J. Am. Chem. Soc. 2003, 125 (26), 7806-7807.

(32) Wehrenberg, B. L.; Yu, D.; Ma, J.; Guyot-Sionnest, P. Conduction in Charged PbSe Nanocrystal Films. J. Phys. Chem. B 2005, 109 (43), 20192-20199.

(33) Boehme, S. C.; Walvis, T. A.; Infante, I.; Grozema, F. C.; Vanmaekelbergh, D.; Siebbeles, L. D. a; Houtepen, A. J. Electrochemical Control over Photoinduced Electron Transfer and Trapping in CdSe-CdTe Quantum-Dot Solids. ACS Nano 2014, 8 (7), 7067-7077.

(34) Boehme, S. C.; Wang, H.; Siebbeles, L. D. a; Vanmaekelbergh, D.; Houtepen, A. J. Electrochemical Charging of CdSe Quantum Dot Films: Dependence on Void Size and Counterion Proximity. ACS Nano 2013, 7 (3), 2500-2508.

(35)

Hickey, S. G.; Riley, D. J. Intensity Modulated Photocurrent Spectroscopy Studies of CdS Nanoparticle Modified Electrodes. Electrochim. Acta 2000, 45 (20), 3277-3282.

(36) Spittel, D.; Poppe, J.; Meerbach, C.; Ziegler, C.; Hickey, S. G.; Eychmüller, A. Absolute 
Energy Level Positions in CdSe Nanostructures from Potential-Modulated Absorption Spectroscopy (EMAS). ACS Nano 2017, 11 (12), 12174-12184.

(37) Miethe, J. F.; Lübkemann, F.; Poppe, J.; Steinbach, F.; Dorfs, D.; Bigall, N. C. Spectroelectrochemical Investigation of the Charge Carrier Kinetics of Gold-Decorated Cadmium Chalcogenide Nanorods. ChemElectroChem 2017, 175-186.

(38) Nádaždy, V.; Schauer, F.; Gmucová, K. Energy Resolved Electrochemical Impedance Spectroscopy for Electronic Structure Mapping in Organic Semiconductors. Appl. Phys. Lett. 2014, 105 (14), 142109.

(39) Schauer, F.; Tkáčová, M.; Nadáždy, V.; Gmucová, K.; Ožvoldová, M.; Tkáč, L.; Chlpík, J. Electronic Structure of UV Degradation Defects in Polysilanes Studied by Energy Resolved - Electrochemical Impedance Spectroscopy. Polym. Degrad. Stab. 2016, 126, 204-208.

(40) Lewis, N. S. Progress in Understanding Electron-Transfer Reactions at Semiconductor/Liquid Interfaces. J. Phys. Chem. B 1998, 102 (25), 4843-4855.

(41) Yazdani, N.; Bozyigit, D.; Vuttivorakulchai, K.; Luisier, M.; Wood, V. Influence of the Surface of a Nanocrystal on Its Electronic and Phononic Properties. arXiv Prepr. 2016, 1611.09930.

(42) Zherebetskyy, D.; Scheele, M.; Zhang, Y.; Bronstein, N.; Thompson, C.; Britt, D.; Salmeron, M.; Alivisatos, P.; Wang, L.-W. Hydroxylation of the Surface of PbS Nanocrystals Passivated with Oleic Acid. Science. 2014, 346 (6206), 1396-1402. 
(43) Stavrinadis, A.; Rath, A. K.; García De Arquer, F. P.; Diedenhofen, S. L.; Magén, C.; Martinez, L.; So, D.; Konstantatos, G. Heterovalent Cation Substitutional Doping for Quantum Dot Homojunction Solar Cells. Nat. Commun. 2013, 4, 1-7.

(44) Trasatti, S. The "absolute" electrode Potential-the End of the Story. Electrochim. Acta 1990, 35 (1), 269-271.

(45) Trasatti, S. The Absolute Electrode Potential: An Explanatory Note. Pure Appl. Chem. 1986, 58 (7), 955-966.

(46) Kuçur, E.; Bücking, W.; Giernoth, R.; Nann, T. Determination of Defect States in Semiconductor Nanocrystals by Cyclic Voltammetry. J. Phys. Chem. B 2005, 109 (43), 20355-20360.

(47) Amelia, M.; Lincheneau, C.; Silvi, S.; Credi, A. Electrochemical Properties of CdSe and CdTe Quantum Dots. Chem. Soc. Rev. 2012, 41 (17), 5728.

(48) Haram, S. K.; Kshirsagar, A.; Gujarathi, Y. D.; Ingole, P. P.; Nene, O. a.; Markad, G. B.; Nanavati, S. P. Quantum Confinement in CdTe Quantum Dots: Investigation through Cyclic Voltammetry Supported by Density Functional Theory (DFT). J. Phys. Chem. C 2011, 115 (14), 6243-6249.

(49) Inamdar, S. N.; Ingole, P. P.; Haram, S. K. Determination of Band Structure Parameters and the Quasi-Particle Gap of CdSe Quantum Dots by Cyclic Voltammetry. ChemPhysChem 2008, 9 (17), 2574-2579.

(50) Bozyigit, D.; Volk, S.; Yarema, O.; Wood, V. Quantification of Deep Traps in 
Nanocrystal Solids, Their Electronic Properties, and Their Influence on Device Behavior. Nano Lett. 2013, 13 (11), 5284-5288.

(51) Bozyigit, D.; Lin, W. M. M.; Yazdani, N.; Yarema, O.; Wood, V. A Quantitative Model for Charge Carrier Transport, Trapping and Recombination in Nanocrystal-Based Solar Cells. Nat. Commun. 2015, 6, 1-10.

(52) Koole, R.; Liljeroth, P.; De Mello Donegá, C.; Vanmaekelbergh, D.; Meijerink, A. Electronic Coupling and Exciton Energy Transfer in CdTe Quantum-Dot Molecules. J. Am. Chem. Soc. 2006, 128 (32), 10436-10441.

(53) Liu, H.; Zhitomirsky, D.; Hoogland, S.; Tang, J.; Kramer, I. J.; Ning, Z.; Sargent, E. H. Systematic Optimization of Quantum Junction Colloidal Quantum Dot Solar Cells. 2012, $101(15), 151112$.

(54) Luther, J. M.; Pietryga, J. M. Stoichiometry Control in Quantum Dots: A Viable Analog to Impurity Doping of Bulk Materials. ACS Nano 2013, 7 (3), 1845-1849.

(55) Tang, J.; Liu, H.; Zhitomirsky, D.; Hoogland, S.; Wang, X.; Furukawa, M.; Levina, L.; Sargent, E. H. Quantum Junction Solar Cells. Nano Lett. 2012, 12 (9), 4889-4894.

(56) Zhitomirsky, D.; Furukawa, M.; Tang, J.; Stadler, P.; Hoogland, S.; Voznyy, O.; Liu, H.; Sargent, E. H. N-Type Colloidal-Quantum-Dot Solids for Photovoltaics. Adv. Mater. 2012, 24 (46), 6181-6185.

(57) Voznyy, O.; Zhitomirsky, D.; Stadler, P.; Ning, Z.; Hoogland, S.; Sargent, E. H. A Charge-Orbital Balance Picture of Doping in Colloidal Quantum Dot Solids. ACS Nano 
2012, 6 (9), 8448-8455.

(58) Oh, S. J.; Berry, N. E.; Choi, J. H.; Gaulding, E. A.; Paik, T.; Hong, S. H.; Murray, C. B.; Kagan, C. R. Stoichiometric Control of Lead Chalcogenide Nanocrystal Solids to Enhance Their Electronic and Optoelectronic Device Performance. ACS Nano 2013, 7 (3), 24132421.

(59) Mocatta, D.; Cohen, G.; Schattner, J.; Millo, O.; Rabani, E.; Banin, U. Heavily Doped Semiconductor Nanocrystal Quantum Dots. Science. 2011, 332 (6025), 77-81.

(60) Sahu, A.; Kang, M. S.; Kompch, A.; Notthoff, C.; Wills, A. W.; Deng, D.; Winterer, M.; Frisbie, C. D.; Norris, D. J. Electronic Impurity Doping in CdSe Nanocrystals. Nano Lett. 2012, 12 (5), 2587-2594.

(61) Shim, M.; Guyot-Sionnest, P. N-Type Collidal Semiconductor Nanocrystals. Nature 2000, 407 (6807), 981-983.

(62) Guyot-Sionnest, P. Charging Colloidal Quantum Dots by Electrochemistry. Microchim. Acta 2008, 160 (3), 309-314.

(63) Koh, W.; Koposov, A. Y.; Stewart, J. T.; Pal, B. N.; Robel, I.; Pietryga, J. M.; Klimov, V. I. Heavily Doped N-Type PbSe and PbS Nanocrystals Using Ground-State Charge Transfer from Cobaltocene. Sci.Rep. 2013, 3, 2004.

(64) Wu, Y. K.; Ye, H. Q.; Coltman, R. R.; Redman, J. K.; Hirth, J. P.; Rogers, H. C.; Huang, J. C.; Deymier, P. a; Smith, D. J.; Bigot, J.; et al. N -Type Conducting CdSe Nanocrystal Solids. 2003, 300 (May), 1277-1280. 
(65) Papagiorgis, P.; Stavrinadis, A.; Othonos, A.; Konstantatos, G.; Itskos, G. The Influence of Doping on the Optoelectronic Properties of PbS Colloidal Quantum Dot Solids. Sci. Rep. 2016, 6 (November 2015), 1-16.

(66) Patnaik, P. Handbook of Inorganic Chemicals; 2003.

(67) BioLogic Science Instruments: Reference electrodes \& porous glass frits http://www.biologic.net/en/accessories/electrodes/reference-electrodes-porous-glass-frits/ (accessed Dec $15,2017)$.

(68) Weidman, M. C.; Yager, K. G.; Tisdale, W. A. Interparticle Spacing and Structural Ordering in Superlattice Pbs Nanocrystal Solids Undergoing Ligand Exchange. Chem. Mater. 2015, 27 (2), 474-482.

(69) Vandevondele, J.; Krack, M.; Mohamed, F.; Parrinello, M.; Chassaing, T.; Hutter, J. Quickstep: Fast and Accurate Density Functional Calculations Using a Mixed Gaussian and Plane Waves Approach. Comput. Phys. Commun. 2005, 167 (2), 103-128.

(70) Lippert, B. G.; Parrinello, J. H. and M. A Hybrid Gaussian and Plane Wave Density Functional Scheme. Mol. Phys. 1997, 92 (3), 477-488.

(71) Voznyy, O.; Mokkath, J. H.; Jain, A.; Sargent, E. H.; Schwingenschlögl, U. Computational Study of Magic-Size Cdse Clusters with Complementary Passivation by Carboxylic and Amine Ligands. J. Phys. Chem. C 2016, 120 (18), 10015-10019.

(72) Bozyigit, D.; Yazdani, N.; Yarema, M.; Yarema, O.; Lin, W. M. M.; Volk, S.; Vuttivorakulchai, K.; Luisier, M.; Juranyi, F.; Wood, V. Soft Surfaces of Nanomaterials 
Enable Strong Phonon Interactions. Nature 2016, 531 (7596), 618-622.

(73) VandeVondele, J.; Hutter, J. Gaussian Basis Sets for Accurate Calculations on Molecular Systems in Gas and Condensed Phases. J. Chem. Phys. 2007, 127 (11).

(74) Hartwigsen, C.; Goedecker, S.; Hutter, J. Relativistic Separable Dual-Space Gaussian Pseudopotentials from H to Rn. Phys. Rev. B 1998, 58 (7), 3641-3662.

(75) Genovese, L.; Deutsch, T.; Neelov, A.; Goedecker, S.; Beylkin, G. Efficient Solution of Poisson's Equation with Free Boundary Conditions. J. Chem. Phys. 2006, 125 (7), 74105.

(76) Genovese, L.; Deutsch, T.; Goedecker, S. Efficient and Accurate Three-Dimensional Poisson Solver for Surface Problems. J. Chem. Phys. 2007, 127, 54704.

(77) Perdew, J. P. Density Functional Theory and the Band Gap Problem. Int. J. Quantum Chem. 2009, 28, 497-523. 